\title{
Embodied Data and Digital Fabrication: Demonstration with Code and Materials
}

\author{
Scott Kildall \\ Independent artist \\ www.kildall.com \\ scottkildall@gmail.com
}

\section{INTRODUCTION}

Data has tangible consequences in the real world. Accordingly, physical data-visualizations have the potential to engage with the actual effects of the data itself. A data-generated sculpture or art installation is something that people can move around, though or inside of. They experience the dimensionality of data with their own natural perceptual mechanisms. However, creating physical data visualizations presents unique material challenges since these objects exist in stasis, rather than in a virtual space with a guided UX design. In this demonstration, I will present my recent research into producing sculptures from data using my custom software code that creates files for digital fabrication machines.

\section{WHAT DOES DATA LOOK LIKE?}

The overarching question that guides my work is: what does data look like? Referencing architecture, my artwork such as Data Crystals (Figure 2) executes codes that maps, stacks and assembles data "bricks" to form unique digital artifacts. The form of these objects are impossible to predict from the original data-mapping, and the clustering code will produce different variations each time it runs.

Other sculptures remove material through intense kinetic energy. Bad Data (Figure 3) and Strewn Fields (Figure 1) both use the waterjet machine to gouge data into physical material using a highpressure stream of water. The material in this case - aluminum honeycomb panels and stone slabs reacts in adverse ways as it splinters and deforms due to the violence of the machine.

\subsection{Material Expression}

Physical data-visualizations act on materials instead of pixels and so there is a dialogue between the data and its material expression. Data Crystals depict municipal data of San Francisco and have a otherworldly ghostly quality of stacked and intersecting cubes. The data gets served from a web portal and is situated in the urban architecture and so the 3D-printed bricks are an appropriate form of expression.

Bad Data captures data that is "bad" in the shallow sense of the word, rendering datasets such as Internet Data Breaches, Worldwide UFO Sightings or Mass Shootings in the United States. The water from the machine gouges and ruptures aluminum honeycomb material in unpredictable ways, similar to the way data tears apart our social fabric. This material is emblematic of the modern era, as aluminum began to be mass-refined at the end of the 19th century. These datasets exemplify conflicts of our times such as science/heresy and digital security/infiltration.

\subsection{Frozen in Time}

Once created, these sculptures cannot be endlessly altered like screen-based data visualizations. This challenges the artwork to work with fixed data or to consider the effect of capturing a specific moment.

For example, Strewn Fields is a data-visualization of meteorite impact data. When a large asteroid enters the earth's atmosphere, it does so at high velocity of approximately $30,000 \mathrm{~km} /$ hour. Before impact, it breaks up into thousands of small fragments, which are meteorites. Usually they hit our planet in the ocean or at remote locations. The intense energy of the waterjet machine gouges the surface of each stone, mirroring the raw kinetic energy of a planetoid colliding with the surface of the Earth. The static etching captures the act of impact, and survives as an antithetical gesture to the event itself. The actual remnants and debris 
(the meteorites) have been collected, sold and scattered and what remains is just a dataset, which I have translated into a physical form.

\subsection{Formal Challenges to Sculpture}

This sort of "data art" challenges the formal aspects of sculpture. Firstly, machine-generated artwork removes the artist's hand from the work, building upon the legacy of algorithmic artwork by Sol Lewitt and others. Execution of this work is conducted by the stepper motor rather than by gestures of the artist.

Secondly, the input source of data are unknowable forms until they are actually rendered. The patterns are neither mathematic nor random, giving a certain quality of perceptual coherence to the work. Data Crystals: Crime Incidents has 30,000 data points. Using code-based clustering algorithms, it creates forms only recently possible with the combination of digital fabrication and large amounts of data.

\section{CODE}

My sculpture-generation tools are customdeveloped in C++ using Open Frameworks, an open source toolkit. My code repositories are on GitHub (https://github.com/scottkildall). My own software bypasses any conventional modeling package. It can handle very complex geometry, and more importantly doesn't have the "look" that a program such as Rhino/Grasshopper generates.

\subsection{Direct-to-Machine}

My process of data-translation is optimized for specific machines. Data Crystals generate STL files which most 3D printers can read. My code generates PostScript (.ps) files for the waterjet machine. The conversation with the machine itself is direct. During the production and iteration process, once I define the workflow, the refinements proceed quickly. It is optimized, like the machine that creates the artwork.

\subsection{London Layering}

In my demonstration, I will use various open data from London. I focus not on data that I want to to acquire, but rather, data that I can acquire. I will demonstrate a custom build of Data Crystals which shows multiple layers of municipal data, and I will run clustering algorithms to create several Data Crystals for the City of London.

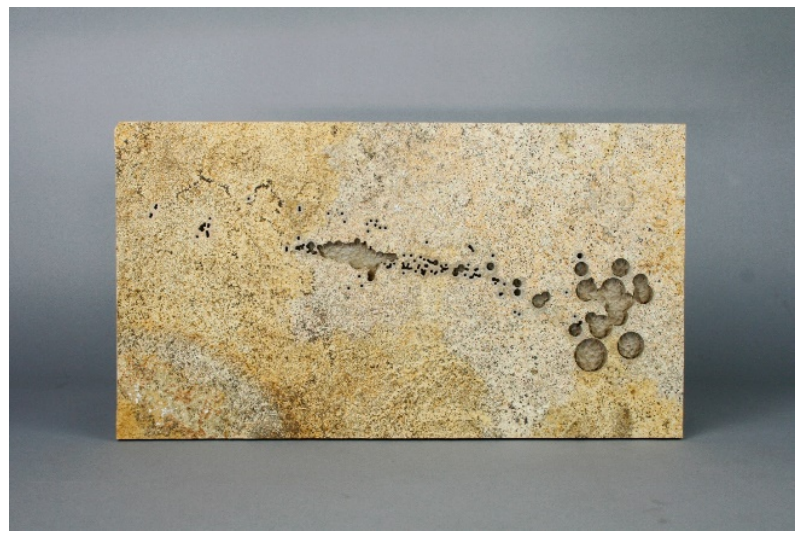

Figure 1: Strewn Fields (2016) by Scott Kildall. Waterjet-etched stone.

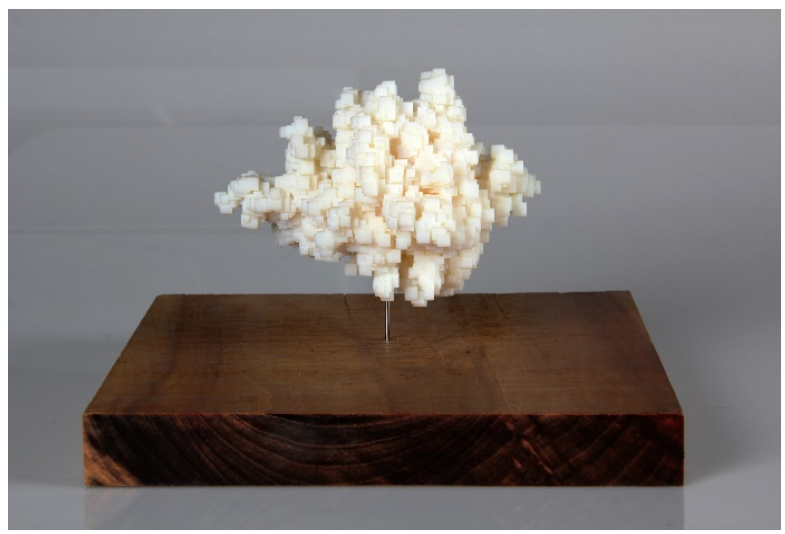

Figure 2: Data Crystals: Crime Incidents (2014) by Scott Kildall. 3D-print mounted on wood.

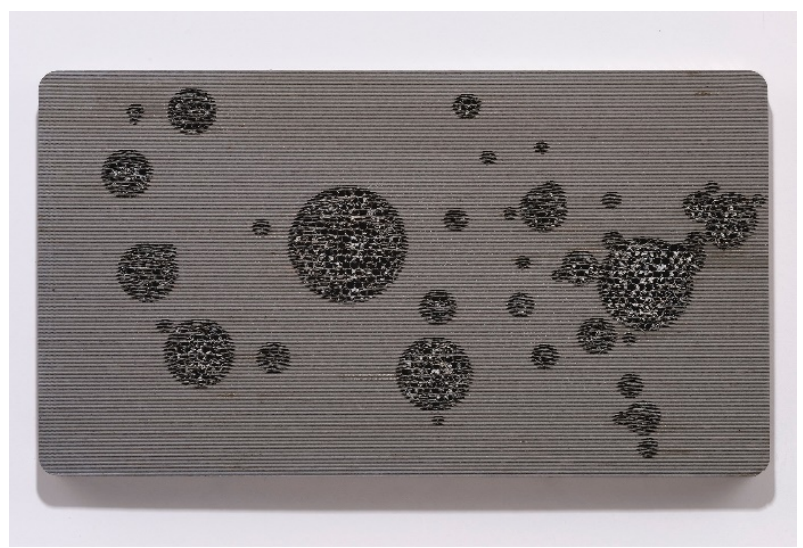

Figure 3: Bad Data: U.S. Mass Shootings (2015) by Scott Kildall.

Waterjet-etched aluminum honeycomb panel. 